\title{
A generalization of Bernstein-Durrmeyer operators on hypercubes by means of an arbitrary measure
}

\author{
Mirella Cappelletti Montano and Vita Leonessa
}

\begin{abstract}
In this paper we introduce and study a sequence of BernsteinDurrmeyer type operators $\left(M_{n, \mu}\right)_{n \geq 1}$, acting on spaces of continuous or integrable functions on the multi-dimensional hypercube $Q_{d}$ of $\mathbf{R}^{d}(d \geq 1)$, defined by means of an arbitrary measure $\mu$. We investigate their approximation properties both in the space of all continuous functions and in $L^{p}$-spaces with respect to $\mu$, also furnishing some estimates of the rate of convergence. Further, we prove an asymptotic formula for the $M_{n, \mu}$ 's. The paper ends with a concrete example.
\end{abstract}

Mathematics Subject Classification (2010): 41A36, 41A63, 41A10.

Keywords: Bernstein operator, Bernstein-Durrmeyer operator, approximation process, asymptotic formula.

\section{Introduction}

Bernstein-Durrmeyer operators were introduced, independently, by Durrmeyer ([15]) and Lupaş ([18]) in their respective dissertations, as a modification of the classical Bernstein operators acting on spaces of integrable functions. More precisely, they are defined by setting

$$
M_{n}(f)(x):=(n+1) \sum_{k=0}^{n}\left(\int_{0}^{1} f(u) p_{n, k}(u) d u\right) p_{n, k}(x),
$$

for every $n \geq 1, x \in[0,1]$ and $f:[0,1] \rightarrow \mathbf{R}$ such that $f p_{n, k} \in L^{1}([0,1])$ for every $k=0, \ldots, n$, where $p_{n, k}(x):=\left(\begin{array}{l}n \\ k\end{array}\right) x^{k}(1-x)^{n-k}$.

These operators were intensively studied by Derriennic ([12]), and during the years they have been subject to many generalizations. The most renowned one is due

This paper has been presented at the fourth edition of the International Conference on Numerical Analysis and Approximation Theory (NAAT 2018), Cluj-Napoca, Romania, September 6-9, 2018. 
to Paltanea ([19], see also $[11,21,22])$, who replaced the weighted measure $p_{n, k} \lambda_{1}$ ( $\lambda_{1}$ being the Lebesgue-Borel measure on $[0,1]$ ) with the absolutely continuous Borel measure with respect to $\lambda_{1}$ with density the normalized Jacobi weights

$$
w_{a, b}(x):=\frac{x^{a}(1-x)^{b}}{\int_{0}^{1} y^{a}(1-y)^{b} d y} \quad a>-1, b>-1, x \in(0,1) .
$$

The Bernstein-Durrmeyer operators with Jacobi weights have been matter of many investigations, in the context of the interval $[0,1]$, in the multi-dimensional framework of simplices $([13,1])$ and, more recently, for hypercubes $([3])$. In particular, in $[1,3]$ the connection between such operators and the study of the so-called FlemingViot differential operators is investigated.

A further step in the possible generalizations of the Bernstein-Durrmeyer operators, which has significant applications in learning theory, consists in replacing the Lebesgue measure with an arbitrary regular Borel measure. This generalization was briefly mentioned by Berens and $\mathrm{Xu}$ in [11] in the context of the interval $[0,1]$, and then intensively studied in $[8,6,7,17,9]$ for the multi-dimensional simplex.

Inspired by these last works, in this paper we introduce and study a sequence of Bernstein-Durrmeyer type operators $\left(M_{n, \mu}\right)_{n \geq 1}$ with respect to an arbitrary measure $\mu$ (see (3.1)-(3.3)), acting both on spaces of continuous and integrable functions on the hypercube $Q_{d}:=[0,1]^{d}$ of $\mathbf{R}^{d}(d \geq 1)$.

First, we prove a necessary and sufficient condition, which involves only properties of the measure $\mu$, in order that the sequence $\left(M_{n, \mu}\right)_{n \geq 1}$ is an approximation process with respect to the uniform norm.

Moreover, following the reasoning in [17] (see also [9]), we show that $\left(M_{n, \mu}\right)_{n \geq 1}$ is an approximation process in $L^{p}\left(Q_{d}, \mu\right)(1 \leq p<+\infty)$ for any Borel measure $\mu$ for which the $M_{n, \mu}$ 's are well defined; this entails that the space $L^{p}\left(Q_{d}, \mu\right)$ is the most natural environment in which studying these operators.

Further, we produce, under suitable conditions, an asymptotic formula for the sequence $\left(M_{n, \mu}\right)_{n \geq 1}$, that involves a second order differential operator.

Finally, a concrete example of Bernstein-Durrmeyer operators on $Q_{d}$ is illustrated.

\section{Notation and preliminary results}

Let us start by fixing some notation. Let $X$ be a compact Hausdorff space. As usual, we denote by $C(X)$ the space of all continuous real-valued functions on $X$. $C(X)$ will be endowed with the uniform norm $\|\cdot\|_{\infty}$, with respect to which it is a Banach space.

A linear operator $T$ on $C(X)$ is called a Markov operator on $C(X)$ if it is positive and $T(\mathbf{1})=\mathbf{1}$, where $\mathbf{1}$ indicates the constant function of constant value 1 on $X$.

If $B_{X}$ is the $\sigma$-algebra of all Borel subsets of $X$, the symbol $M^{+}(X)$ (resp., $\left.M_{b}^{+}(X)\right)$ stands for the cone of all regular Borel measures on $X$ (resp., the cone of all bounded Borel measures on $X)$.

For a measure $\mu \in M^{+}(X)$, we denote by $\operatorname{supp}(\mu)$ the support of $\mu$, i.e., the complement of the largest open subset of $X$ on which $\mu$ is zero. We recall that a 
measure $\mu$ on $X$ is said to be strictly positive on $X$ if $\mu(X \cap A)>0$ for every open subset $A$ such that $A \cap X \neq \emptyset$. We remark that, on the account of [20, Prop. 13, p. 408], $\mu$ is a strictly positive measure if and only if $\operatorname{supp}(\mu)=X$.

Let $\mu \in M^{+}(X)$ and $1 \leq p<+\infty$. As usual, $L^{p}(X, \mu)$ is the space of all (the equivalence classes of) Borel measurable, real-valued functions on $X$ which are $\mu$-integrable in the $p^{\text {th }}$ power. The space $L^{p}(X, \mu)$ is endowed with the norm

$$
\|f\|_{p}:=\left(\int_{X}|f|^{p} d \mu\right)^{1 / p} \quad\left(f \in L^{p}(X, \mu)\right) .
$$

Now, let $\gamma=\left(\gamma_{1}, \ldots, \gamma_{d}\right) \in \mathbf{R}^{d}, d \geq 1$. If $x=\left(x_{1}, \ldots, x_{d}\right) \in \mathbf{R}^{d}, x_{i}>0$ for every $i=1, \ldots, d$, we set

$$
x^{\gamma}:=\prod_{i=1}^{d} x_{i}^{\gamma_{i}} .
$$

If $\gamma_{i} \geq 0$ and $x_{i} \geq 0$ for each $i=1, \ldots, d$, then $x^{\gamma}$ is similarly defined as above assuming $0^{0}:=1$.

If $x=\left(x_{1}, \ldots, x_{d}\right), y=\left(y_{1}, \ldots, y_{d}\right) \in \mathbf{R}^{d}$, then we write $x \leq y$ whenever $x_{i} \leq y_{i}$ for every $i=1, \ldots, d$.

Let $j=\left(j_{1}, \ldots, j_{d}\right), k=\left(k_{1}, \ldots, k_{d}\right) \in \mathbf{N}^{d}$ be two multi-indices such that $k \leq j$; we define

$$
\left(\begin{array}{l}
j \\
k
\end{array}\right):=\prod_{i=1}^{d}\left(\begin{array}{l}
j_{i} \\
k_{i}
\end{array}\right) .
$$

We also set $0_{d}:=(0, \ldots, 0)$ and, for every $n \geq 1, n_{d}:=(n, \ldots, n)$.

All the results of this paper concern the case where $X$ is the $d$-dimensional hypercube $Q_{d}:=[0,1]^{d}, d \geq 1$.

First, for each $i=1, \ldots, d$, the symbol $p r_{i}$ stands for the $i$-th coordinate function on $Q_{d}$, which is defined by setting $\operatorname{pr}_{i}(x)=x_{i}$ for every $x \in Q_{d}$.

Moreover, let us consider the partition $P=\left\{\xi_{0}, \xi_{1}, \xi_{2}\right\}$ of the unit interval $[0,1]$ such that $\xi_{0}=0, \xi_{1}=\frac{1}{2}$ and $\xi_{2}=1$. Then, $P$ yields a partition of $Q_{d}$ composed by closed sub-hypercubes of the form

$$
Q_{d, j}:=\left[\xi_{j_{1}}, \xi_{j_{1}+1}\right] \times \cdots \times\left[\xi_{j_{d}}, \xi_{j_{d}+1}\right]
$$

where $j=\left(j_{1}, \ldots, j_{d}\right)$ is such that $j_{i}=0$ or $j_{i}=1$ for every $i=1, \ldots, d$ (briefly, $\left.j \in\{0,1\}^{d}\right)$.

For $a \in Q_{d}$ and $r>0$, we define the open $d$-dimensional hypercube

$$
I_{d}(a ; r):=\left(a_{1}-r, a_{1}+r\right) \times \cdots \times\left(a_{d}-r, a_{d}+r\right),
$$

and the closed $d$-dimensional hyperrectangle

$$
J_{d}(a ; r):=\left[a_{1}, a_{1}+r\right] \times \cdots \times\left[a_{d}, a_{d}+r\right] .
$$

Remark 2.1. Observe that, if $a$ belongs to some $Q_{d, j}$ and if $r \leq \frac{1}{2}$, then $J_{d}(a ; r) \subset Q_{d}$. Indeed, $a \in Q_{d, j}$ means that $\xi_{j_{i}} \leq a_{i} \leq \xi_{j_{i}+1}$ for every $i=1, \ldots, d$. Hence, if $x \in J_{d}(a ; r)$, for any $i=1, \ldots, d, x_{i} \geq 0$, since $j_{i} \in\left\{0, \frac{1}{2}, 1\right\}$. Now, fix $i=1, \ldots, d$ and suppose that $j_{i}=0$; in this case $0 \leq a_{i} \leq \frac{1}{2}$ from which it easily follows $x_{i} \leq 1$. 
Otherwise, if $j_{i}=1, \frac{1}{2} \leq a_{i} \leq 1$, that is $0 \leq 1-a_{i} \leq \frac{1}{2}$, therefore $a_{i}+r \leq 1$ is equivalent to the true inequality $r \leq 1-a_{i} \leq \frac{1}{2}$, and again $x_{i} \leq 1$.

The following lemma will play an important role in the next section. It deals with the case of the unit interval $[0,1]$.

Lemma 2.2. Let $0<r \leq \frac{1}{2}$. Then, there exists a positive constant $K=K(r)$, depending only on $r$, such that

$$
\frac{\max _{x \in[0,1] \backslash(a-r, a+r)} x^{a}(1-x)^{1-a}}{\min _{x \in\left[a, a+r^{2}\right]} x^{a}(1-x)^{1-a}}<K<1 .
$$

Proof. The proof can be found in [6, Lemma 3 and Lemma 4] (see also [7, p. 738]). In particular it is worth noticing that

$$
\max _{x \in[0,1] \backslash(a-r, a+r)} x^{a}(1-x)^{1-a}= \begin{cases}(a+r)^{a}(1-a-r)^{1-a} & \text { if } 0 \leq a \leq \frac{1}{2}, \\ (a-r)^{a}(1-a+r)^{1-a} & \text { if } \frac{1}{2} \leq a \leq 1,\end{cases}
$$

and

$$
\min _{x \in\left[a, a+r^{2}\right]} x^{a}(1-x)^{1-a}=\left(a+r^{2}\right)^{a}\left(1-a-r^{2}\right)^{1-a} .
$$

Finally, coming back to the case $d \geq 1$, we state the following result.

Lemma 2.3. Consider $\mu \in M_{b}^{+}\left(Q_{d}\right)$ such that $\operatorname{supp}(\mu)=Q_{d}$. Then, for every $r>0$ and $j \in\{0,1\}^{d}$,

$$
\inf _{a \in Q_{d, j}} \mu\left(J_{d}(a ; r)\right)>0,
$$

where $Q_{d, j}$ and $J_{d}(a ; r)$ are defined, respectively, by (2.1) and (2.3).

Proof. We begin with supposing $r \leq 1 / 2$. In this case, from Remark 2.1 it follows that the interior $\stackrel{\circ}{J}_{d}(a ; r)$ of $J_{d}(a ; r)$ is contained into $Q_{d}$, so that $\stackrel{\circ}{J}_{d}(a ; r)=\stackrel{\circ}{J}_{d}(a ; r) \cap Q_{d}$. Since $\mu$ is strictly positive on $Q_{d}$, we have that

$$
0<\mu\left(\stackrel{\circ}{J}_{d}(a ; r) \cap Q_{d}\right)=\mu\left(\stackrel{\circ}{J}_{d}(a ; r)\right) \leq \mu\left(J_{d}(a ; r)\right) .
$$

The case $r>1 / 2$ is an easy consequence of the fact that $\mu\left(J_{d}(a ; r)\right) \geq \mu\left(J_{d}\left(a ; \frac{1}{2}\right)\right)>0$, where the last inequality is true for the first part of the proof.

On account of Lemma 2.3, we set

$$
C(\mu, r):=\min _{j \in\{0,1\}^{d}} \inf _{a \in Q_{d, j}} \mu\left(J_{d}(a ; r)\right)>0 .
$$




\section{Bernstein-Durrmeyer operators on $Q_{d}$ with respect to arbitrary measures}

Let $\mu \in M_{b}^{+}\left(Q_{d}\right)$ be a nonnegative Borel measure on $Q_{d}$ satisfying the assumption

$$
\operatorname{supp}(\mu) \backslash \partial Q_{d} \neq \emptyset \text {. }
$$

For every $n \geq 1$, let us consider the operator $M_{n, \mu}: L^{1}\left(Q_{d}, \mu\right) \rightarrow C\left(Q_{d}\right)$ defined by setting, for every $f \in L^{1}\left(Q_{d}, \mu\right)$ and $x \in Q_{d}$,

$$
M_{n, \mu}(f)(x):=\sum_{\substack{h \in \mathbf{N}^{d} \\
0_{d} \leq h \leq n_{d}}} \omega_{n_{d}, h}(f, \mu)\left(\begin{array}{c}
n_{d} \\
h
\end{array}\right) x^{h}\left(1_{d}-x\right)^{n_{d}-h},
$$

where, for every $n \geq 1$ and $h=\left(h_{1}, \ldots h_{d}\right) \in \mathbf{N}^{d}, 0_{d} \leq h \leq n_{d}$,

$$
\omega_{n_{d}, h}(f, \mu):=\frac{1}{\int_{Q_{d}} y^{h}\left(1_{d}-y\right)^{n_{d}-h} d \mu(y)} \int_{Q_{d}} y^{h}\left(1_{d}-y\right)^{n_{d}-h} f(y) d \mu(y) .
$$

We remark that assumption (3.1) guarantees that, for every $n \geq 1$ and $h \in \mathbf{N}^{d}$, $0_{d} \leq h \leq n_{d}$

$$
\int_{Q_{d}} y^{h}\left(1_{d}-y\right)^{n_{d}-h} d \mu(y)>0 .
$$

Clearly, the operators $M_{n, \mu}$ are linear, positive, and $M_{n, \mu}(\mathbf{1})=\mathbf{1}$, so that the restriction of each $M_{n, \mu}$ to $C\left(Q_{d}\right)$ is a Markov operator on $C\left(Q_{d}\right)$ with unitary norm. Moreover, for any $f \in L^{1}\left(Q_{d}, \mu\right)$ and $n \geq 1, M_{n, \mu}(f)$ is a polynomial of total degree at most $n$.

In order to discuss the convergence of the sequence $\left(M_{n, \mu}\right)_{n \geq 1}$ both on $C\left(Q_{d}\right)$ and $L^{p}\left(Q_{d}, \mu\right)(p \geq 1)$, first of all, we recall the definition of the classical Bernstein operators on $Q_{d}$ (see [16] and the references therein). They are defined by setting, for any $n \geq 1, f \in C\left(Q_{d}\right)$, and $x \in Q_{d}$,

$$
B_{n}(f)(x)=\sum_{\substack{h \in \mathbf{N}^{d} \\
0_{d} \leq h \leq n_{d}}} f\left(\frac{h}{n}\right)\left(\begin{array}{c}
n_{d} \\
h
\end{array}\right) x^{h}\left(1_{d}-x\right)^{n_{d}-h} .
$$

The sequence $\left(B_{n}\right)_{n \geq 1}$ is an approximation process on $C\left(Q_{d}\right)$, i.e., for any $f \in C\left(Q_{d}\right)$

$$
\lim _{n \rightarrow \infty} B_{n}(f)=f \quad \text { uniformly on } Q_{d} \text {. }
$$

Observe in particular that $B_{n}(\mathbf{1})=\mathbf{1}$, or equivalently, that

$$
\sum_{\substack{h \in \mathbf{N}^{d} \\
0_{d} \leq h \leq n_{d}}}\left(\begin{array}{c}
n_{d} \\
h
\end{array}\right) x^{h}\left(1_{d}-x\right)^{n_{d}-h}=1 \quad \text { for every } \quad x \in Q_{d} .
$$

Finally, we have that, for every $n \geq 1$ and $i=1, \ldots, d$,

$$
B_{n}\left(p r_{i}\right)=p r_{i} \quad \text { and } \quad B_{n}\left(p r_{i}^{2}\right)=\frac{1}{n} p r_{i}+\frac{n-1}{n} p r_{i}^{2} .
$$




\subsection{Approximation properties on $C\left(Q_{d}\right)$}

In what follows we study the convergence properties of the sequence $\left(M_{n, \mu}(f)\right)_{n \geq 1}$ on the space $C\left(Q_{d}\right)$.

Theorem 3.1. The following statements are equivalent:

(i) For every $f \in C\left(Q_{d}\right)$,

$$
\lim _{n \rightarrow \infty} M_{n, \mu}(f)=f \quad \text { uniformly on } Q_{d} .
$$

(ii) $\operatorname{supp}(\mu)=Q_{d}$.

Proof. $(i) \Longrightarrow($ ii $)$. Suppose that there exists a nonempty open set $A \subset Q_{d}$ such that $\mu(A)=0$. Then, for every $f \in C\left(Q_{d}\right), f=0$ on $Q_{d} \backslash A$, on account of (3.5) for operators $B_{n}$, we have

$$
\int_{Q_{d}} y^{h}\left(1_{d}-y\right)^{n_{d}-h} f(y) d \mu(y)=\left\{\int_{A}+\int_{Q_{d} \backslash A}\right\} y^{h}\left(1_{d}-y\right)^{n_{d}-h} f(y) d \mu(y)=0 .
$$

Therefore, $M_{n, \mu}(f)=0$ and this leads to a contradiction because of $(i)$.

$($ ii $) \Longrightarrow(i)$. For $n \geq 1, f \in C\left(Q_{d}\right)$ and $x \in Q_{d}$, we have

$$
\begin{aligned}
& \left|M_{n, \mu}(f)(x)-f(x)\right| \leq\left|M_{n, \mu}(f)(x)-B_{n}(f)(x)\right|+\left|B_{n}(f)(x)-f(x)\right| \\
& \leq \sum_{\substack{h \in \mathbf{N}^{d} \\
0_{d} \leq h \leq n}}\left|\omega_{n_{d}, h}(f, \mu)-f\left(\frac{h}{n_{d}}\right)\right|\left(\begin{array}{c}
n_{d} \\
h
\end{array}\right) x^{h}\left(1_{d}-x\right)^{n_{d}-h}+\left\|B_{n}(f)-f\right\|_{\infty} \\
& \leq \max _{\substack{h \in \mathbf{N}^{d} \\
0_{d} \leq h \leq n}}\left|\omega_{n_{d}, h}(f, \mu)-f\left(\frac{h}{n_{d}}\right)\right|+\left\|B_{n}(f)-f\right\|_{\infty},
\end{aligned}
$$

as (3.6) holds true. Thus, keeping (3.5) in mind, in order to get the claim it is sufficient to prove that

$$
\lim _{n \rightarrow \infty} \max _{\substack{h \in \mathbf{N}^{d} \\ 0_{d} \leq h \leq n_{d}}}\left|\omega_{n_{d}, h}(f, \mu)-f\left(\frac{h}{n}\right)\right|=0 .
$$

We begin to observing that, since $f$ is uniformly continuous on $Q_{d}$,

$$
\begin{aligned}
& \text { for a fixed } \varepsilon>0, \text { there exists } \tilde{\delta}>0 \text { such that, for every } \\
& x, y \in Q_{d} \text { with }\|x-y\|<\tilde{\delta} \text {, then }|f(x)-f(y)|<\varepsilon
\end{aligned}
$$

where by $\|\cdot\|$ we indicate the $l^{\infty}$-norm on $\mathbf{R}^{d}$ defined by setting $\|x\|:=\max _{i=1, \ldots, d}\left|x_{i}\right|$ $\left(x \in \mathbf{R}^{d}\right)$.

Fix $\delta=\min \left\{\tilde{\delta}, \frac{1}{2}\right\}, n \geq 1, h \in \mathbf{N}^{d}, 0_{d} \leq h \leq n_{d}$, and $j \in\{0,1\}^{d}$ such that, the point $\frac{h}{n}$ belongs to the piece $Q_{d, j}$ of $Q_{d}$ (see (2.1)).

Then

$$
\left|\omega_{n_{d}, h}(f, \mu)-f\left(\frac{h}{n}\right)\right| \leq \frac{\int_{Q_{d}}\left|f(y)-f\left(\frac{h}{n}\right)\right| y^{h}\left(1_{d}-y\right)^{n_{d}-h} d \mu(y)}{\int_{Q_{d}} y^{h}\left(1_{d}-y\right)^{n_{d}-h} d \mu(y)} .
$$


Now, rewrite $Q_{d}=\left(Q_{d} \cap I_{d}\left(\frac{h}{n} ; \delta\right)\right) \cup\left(Q_{d} \backslash I_{d}\left(\frac{h}{n} ; \delta\right)\right)$ (see (2.2)), and observe that, from (3.10) it follows that $\left|f(y)-f\left(\frac{h}{n}\right)\right|<\varepsilon$ when $y \in Q_{d} \cap I_{d}\left(\frac{h}{n} ; \delta\right)$. Hence, setting $M:=\|f\|_{\infty}$, from Remark 2.1, (2.3) and (2.4), we get

$$
\begin{aligned}
& \left|\omega_{n_{d}, h}(f, \mu)-f\left(\frac{h}{n}\right)\right| \leq \varepsilon+2 M \frac{\int_{Q_{d} \backslash I_{d}\left(\frac{h}{n} ; \delta\right)} y^{h}\left(1_{d}-y\right)^{n_{d}-h} d \mu(y)}{\int_{Q_{d}} y^{h}\left(1_{d}-y\right)^{n_{d}-h} d \mu(y)} \\
& <\varepsilon+2 M \frac{\int_{Q_{d} \backslash I_{d}\left(\frac{h}{n} ; \delta\right)} y^{h}\left(1_{d}-y\right)^{n_{d}-h} d \mu(y)}{\int_{J_{d}\left(\frac{h}{n} ; \delta^{2}\right)} y^{h}\left(1_{d}-y\right)^{n_{d}-h} d \mu(y)} \\
& <\varepsilon+2 M \frac{\mu\left(Q_{d}\right)}{\mu\left(J_{d}\left(\frac{h}{n} ; \delta^{2}\right)\right)} \frac{\max _{y \in Q_{d} \backslash I_{d}\left(\frac{h}{n} ; \delta\right)} y^{h}\left(1_{d}-y\right)^{n_{d}-h}}{\min _{y \in J_{d}\left(\frac{h}{n} ; \delta^{2}\right)} y^{h}\left(1_{d}-y\right)^{n_{d}-h}} \\
& <\varepsilon+2 M \frac{\mu\left(Q_{d}\right)}{C\left(\mu, \delta^{2}\right)} \frac{\left(\max _{y \in Q_{d} \backslash I_{d}(a ; \delta)} y^{a}\left(1_{d}-y\right)^{1_{d}-a}\right)^{n}}{\left(\min _{y \in J_{d}\left(a ; \delta^{2}\right)} y^{a}\left(1_{d}-y\right)^{1_{d}-a}\right)^{n}}
\end{aligned}
$$

where in the last inequality $a:=\frac{h}{n}$.

Since

$$
\max _{y \in Q_{d} \backslash I_{d}(a ; \delta)} y^{a}\left(1_{d}-y\right)^{1_{d}-a}=\prod_{i=1}^{d} \max _{y_{i} \in[0,1] \backslash\left(a_{i}-\delta, a_{i}+\delta\right)} y_{i}^{a_{i}}\left(1-y_{i}\right)^{1-a_{i}},
$$

and

$$
\min _{y \in J_{d}\left(a ; \delta^{2}\right)} y^{a}\left(1_{d}-y\right)^{1_{d}-a}=\prod_{i=1}^{d} \min _{y_{i} \in\left[a_{i}, a_{i}+\delta^{2}\right]} y_{i}^{a_{i}}\left(1-y_{i}\right)^{1-a_{i}},
$$

by applying Lemma 2.2 we get that

$$
\frac{\left(\max _{y \in Q_{d} \backslash I_{d}(a ; \delta)} y^{a}\left(1_{d}-y\right)^{1_{d}-a}\right)^{n}}{\left(\min _{y \in J_{d}\left(a ; \delta^{2}\right)} y^{a}\left(1_{d}-y\right)^{1_{d}-a}\right)^{n}}<K(r)^{n} \rightarrow 0 \quad \text { as } n \rightarrow \infty .
$$

Therefore, there exists $n_{\varepsilon} \in \mathbf{N}$ such that, for every $n \geq n_{\varepsilon},(K(r))^{n}<\varepsilon \frac{C\left(\mu, \delta^{2}\right)}{2 M \mu\left(Q_{d}\right)}$. Accordingly, for every $n \geq n_{\epsilon}$ and $h \in \mathbf{N}^{d}, 0_{d} \leq h \leq n_{d}$,

$$
\left|\omega_{n_{d}, h}(f, \mu)-f\left(\frac{h}{n}\right)\right|<2 \varepsilon
$$

and this completes the proof of (3.9).

At the end of Section 4 we present, under suitable assumptions, an estimate of the convergence in (3.8).

\subsection{Approximation properties on $L^{p}\left(Q_{d}, \mu\right)$}

In this section we are interested in the convergence properties of the BernsteinDurrmeyer operators $M_{n, \mu}$ defined by (3.1)-(3.3) in the space $L^{p}\left(Q_{d}, \mu\right)(1 \leq p<+\infty)$.

First note that, if $n \geq 1$ and $f \in L^{1}\left(Q_{d}, \mu\right)$, we get

$$
\int_{Q_{d}} M_{n, \mu}(f) d \mu=\int_{Q_{d}} f d \mu \text {. }
$$


Indeed, keeping (3.6) in mind,

$$
\begin{aligned}
& \int_{Q_{d}} M_{n, \mu}(f) d \mu=\sum_{\substack{h \in \mathbf{N}^{d} \\
0_{d} \leq h \leq n_{d}}} \omega_{n_{d}, h}(f, \mu)\left(\begin{array}{c}
n_{d} \\
h
\end{array}\right) \int_{Q_{d}} x^{h}\left(1_{d}-x\right)^{n_{d}-h} d \mu(x) \\
& =\sum_{\substack{h \in \mathbf{N}^{d} \\
0_{d} \leq h \leq n_{d}}}\left(\begin{array}{c}
n_{d} \\
h
\end{array}\right) \int_{Q_{d}} y^{h}\left(1_{d}-y\right)^{n_{d}-h} f(y) d \mu(y) \\
& =\int_{Q_{d}} \sum_{\substack{h \in \mathbf{N}^{d} \\
0_{d} \leq h \leq n_{d}}}\left[\left(\begin{array}{c}
n_{d} \\
h
\end{array}\right) y^{h}\left(1_{d}-y\right)^{n_{d}-h}\right] f(y) d \mu(y)=\int_{Q_{d}} f(y) d \mu(y) .
\end{aligned}
$$

Moreover, by using the convexity of the function $|t|^{p}(t \in \mathbf{R})$ and (3.6), for any $n \geq 1$ and $f \in L^{p}\left(Q_{d}, \mu\right)$, we obtain

$$
\left|M_{n, \mu}(f)(x)\right|^{p} \leq \sum_{\substack{h \in \mathbf{N}^{d} \\
0_{d} \leq h \leq n_{d}}}\left(\omega_{n_{d}, h}(|f|, \mu)\right)^{p}\left(\begin{array}{c}
n_{d} \\
h
\end{array}\right) x^{h}\left(1_{d}-x\right)^{n_{d}-h} .
$$

Now, by applying the integral Jensen inequality (see, e.g. [5]) to the probability measure $\rho$ on $Q_{d}$ which is absolutely continuous with respect to $\mu$ with density the weight function $w_{n_{d}, h}$ defined on the interior $\stackrel{\circ}{Q}_{d}$ of $Q_{d}$ as

$$
w_{n_{d}, h}(x)=\frac{x^{h}\left(1_{d}-x\right)^{n_{d}-h}}{\int_{Q_{d}} y^{h}\left(1_{d}-y\right)^{n_{d}-h} d \mu(y)} \quad\left(x \in \stackrel{\circ}{Q_{d}}\right),
$$

we get (see (3.3))

$$
\left(\omega_{n_{d}, h}(|f|, \mu)\right)^{p}=\left(\int_{Q_{d}}|f(y)| d \rho(y)\right)^{p} \leq \int_{Q_{d}}|f(y)|^{p} d \rho(y)=\omega_{n_{d}, h}\left(|f|^{p}, \mu\right),
$$

and hence, $\left|M_{n, \mu}(f)\right|^{p} \leq M_{n, \mu}\left(|f|^{p}\right)$. Therefore, by integrating with respect to $\mu$ over $Q_{d}$, we gain

$$
\int_{Q_{d}}\left|M_{n, \mu}(f)\right|^{p} d \mu \leq \int_{Q_{d}} M_{n, \mu}\left(|f|^{p}\right) d \mu=\int_{Q_{d}}|f|^{p} d \mu,
$$

where in the last equality we have used (3.11). Inequality (3.12) means that $M_{n, \mu}$ maps $L^{p}\left(Q_{d}, \mu\right)$ into itself and, in particular that each restriction $\left.M_{n}\right|_{L^{p}\left(Q_{d}, \mu\right)}$ coincides with the extension of $\left.M_{n}\right|_{C\left(Q_{d}\right)}$ to $L^{p}\left(Q_{d}, \mu\right)$.

Thanks to these considerations, we are able to get the following result.

Proposition 3.2. Consider $\mu \in M_{b}^{+}\left(Q_{d}\right)$ satisfying (3.1). Then, for every $n \geq 1$ and $1 \leq p<+\infty$, for the operator $M_{n, \mu}: L^{p}\left(Q_{d}, \mu\right) \rightarrow L^{p}\left(Q_{d}, \mu\right)$ the following inequality holds:

$$
\left\|M_{n, \mu}(f)\right\|_{L^{p}} \leq\|f\|_{L^{p}} \quad\left(f \in L^{p}\left(Q_{d}, \mu\right)\right) .
$$

Property (ii) on $\mu$ for the convergence in $C\left(Q_{d}\right)$ seems to be too strong for spaces of integrable functions, and in fact, following the idea used by $\mathrm{Li}$ in the case of simplices (see [17]; see also [9]), we prove that $\left(M_{n, \mu}\right)_{n \geq 1}$ constitutes a positive approximation process in $L^{p}\left(Q_{d}, \mu\right)$ requiring only that $\mu$ satisfies (3.1). 
We need some additional notation. Consider the space $C^{1}\left(Q_{d}\right)$ of all real-valued continuous functions on $Q_{d}$ which are continuously differentiable on $\stackrel{\circ}{Q}_{d}$ and whose partial derivatives can be continuously extended to $Q_{d}$. We shall continue to denote by $\frac{\partial}{\partial x_{i}}$ the continuous extensions to $Q_{d}$ of $\frac{\partial}{\partial x_{i}}$. Moreover, the space $C^{1}\left(Q_{d}\right)$ will be equipped with the seminorm $\|\mid \nabla g\|\left\|:=\max _{i=1, \ldots, d}\right\| \frac{\partial}{\partial x_{i}} g \|_{\infty}$.

Further, let $\mathcal{K}(f, t)_{p}$ be the $\mathcal{K}$-functional (see, e.g. [14]) defined by

$$
\mathcal{K}(f, t)_{p}=\inf _{g \in C^{1}\left(Q_{d}\right)}\left\{\|f-g\|_{L^{p}}+t \mid\|\nabla g\| \|\right\} \quad \text { for } p \geq 1, t>0 .
$$

In particular, for every $f \in L^{p}\left(Q_{d}, \mu\right)$, one has

$$
\mathcal{K}(f, t)_{p} \rightarrow 0 \quad \text { as } t \rightarrow 0 .
$$

Theorem 3.3. Let $\mu \in M_{b}^{+}\left(Q_{d}\right)$ satisfying (3.1) and consider the operators $M_{n, \mu}$ defined by (3.2). For every $f \in L^{p}\left(Q_{d}, \mu\right), 1 \leq p<+\infty$ and $n \geq 1$,

$$
\left\|M_{n, \mu}(f)-f\right\|_{p} \leq 2 \mathcal{K}\left(f, C_{p}\left(\mu\left(Q_{d}\right)\right)^{\frac{1}{p}} n^{-\frac{1}{2}}\right)_{p}
$$

where $C_{p}$ is a constant depending only on $p$ and $d$. In particular,

$$
\lim _{n \rightarrow \infty} M_{n, \mu}(f)=f \quad \text { in } L^{p}\left(Q_{d}, \mu\right) .
$$

Proof. By an inspection of Theorems 2.1 and 2.2 in [17], one notes that the arguments used there work also for hypercubes. In fact, first we have that, for any $n \geq 1$, $f \in L^{p}\left(Q_{d}, \mu\right)$ and $p \geq 1$, the following estimate holds:

$$
\left\|M_{n, \mu}(f)-f\right\|_{p} \leq 2 \mathcal{K}\left(f, \Delta_{n, p} / 2\right)_{p}
$$

where

$$
\begin{aligned}
\Delta_{n, p} & :=\sum_{i=1}^{d}\left(\int_{Q_{d}}\left|M_{n, \mu}\left(\left|p r_{i}-p r_{i}(x) \mathbf{1}\right|\right)(x)\right|^{p} d \mu(x)\right)^{1 / p} \\
& =\sum_{i=1}^{d}\left\|M_{n, \mu}\left(\left|p r_{i}-p r_{i}(x) \mathbf{1}\right|\right)\right\|_{L^{p}} .
\end{aligned}
$$

The proof of (3.17) runs as in [17, Theorem 2.1] (see also [8, Theorem 4.5]) on account of (3.13) and the well-known equivalence between $l^{\infty}$-norm and $l^{1}$-norm in $\mathbf{R}^{d}$.

Subsequently, estimates of $\Delta_{n, p}$ similar to those in [9, Theorem 1.1, Lemma 1.1] can be obtained, thanks to (3.6) and, moreover, to the fact that the expressions of $B_{n}\left(p r_{i}\right)$ and $B_{n}\left(p r_{i}^{2}\right)$ in the case of hypercubes (see (3.7)) are the same as those for simplices (see, e.g [17, Lemma 2.1]). In particular, for every $i=1, \ldots, d$,

$$
\left\|M_{n, \mu}\left(\left|p r_{i}-p r_{i}(x) \mathbf{1}\right|\right)\right\|_{L^{p}} \leq c_{p}\left(\mu\left(Q_{d}\right)\right)^{\frac{1}{p}} n^{-\frac{1}{2}}
$$

where $c_{p}$ is a constant depending only on $p$. Hence,

$$
\left\|M_{n, \mu}(f)-f\right\|_{p} \leq 2 \mathcal{K}\left(f, d c_{p}\left(\mu\left(Q_{d}\right)\right)^{\frac{1}{p}} n^{-\frac{1}{2}} / 2\right)_{p},
$$

which leads to (3.15) and, letting $n \rightarrow \infty$, we get (3.16) by virtue of (3.14). 


\section{Asymptotic formula for the operators $M_{n, \mu}$}

In order to present an asymptotic formula for the operators $M_{n, \mu}$, we need some further notation.

In particular, we denote by $C^{2}\left(Q_{d}\right)$ the space of all real-valued continuous functions on $Q_{d}$ which are twice-continuously differentiable on $\stackrel{\circ}{Q}_{d}$ and whose partial derivatives up to the order 2 can be continuously extended to $Q_{d}$. We shall continue to indicate by $\frac{\partial}{\partial x_{i}}$ and $\frac{\partial^{2}}{\partial x_{i} \partial x_{j}}$ the continuous extensions to $Q_{d}$ of $\frac{\partial}{\partial x_{i}}$ and $\frac{\partial^{2}}{\partial x_{i} \partial x_{j}}$. Moreover, for every $x \in Q_{d}$, we denote by $\Psi_{x}, d_{x} \in C\left(Q_{d}\right)$ the functions defined by

$$
\Psi_{x}(y):=y-x \quad \text { and } \quad d_{x}(y):=\left(\sum_{i=1}^{d}\left|y_{i}-x_{i}\right|^{2}\right)^{1 / 2} \quad\left(y \in Q_{d}\right) .
$$

We notice that, for every $y=\left(y_{1}, \ldots, y_{d}\right) \in Q_{d}$, and $i=1, \ldots, d$,

$$
\left(p r_{i} \circ \Psi_{x}\right)(y)=p r_{i}(y-x)=y_{i}-x_{i}=p r_{i}(y)-x_{i} ;
$$

accordingly,

$$
d_{x}^{2}=\sum_{i=1}^{d}\left(p r_{i} \circ \Psi_{x}\right)^{2} \quad \text { and } \quad d_{x}^{4}=\sum_{i, j=1}^{d}\left(p r_{i} \circ \Psi_{x}\right)^{2}\left(p r_{j} \circ \Psi_{x}\right)^{2} .
$$

Theorem 4.1. Under the hypothesis $\operatorname{supp}(\mu)=Q_{d}$, assume also that:

(i) For every $i=1, \ldots, d$, there exists $\beta_{i} \in C\left(Q_{d}\right)$ such that

$$
\lim _{n \rightarrow \infty} n\left(M_{n, \mu}\left(p r_{i}\right)-p r_{i}\right)=\beta_{i} \quad \text { uniformly on } Q_{d} .
$$

(ii) For every $i, j=1, \ldots, d$, there exists $\gamma_{i j} \in C\left(Q_{d}\right)$ such that

$$
\lim _{n \rightarrow \infty} n\left(M_{n, \mu}\left(p r_{i} p r_{j}\right)-p r_{i} p r_{j}\right)=\gamma_{i j} \quad \text { uniformly on } Q_{d} \text {. }
$$

(iii) For every $x \in Q_{d}, n \geq 1$ and for every $h \in \mathbf{N}^{d}, 0_{d} \leq h \leq n_{d}$, one has

$$
\lim _{n \rightarrow \infty} n \max _{\substack{h \in \mathbf{N}^{d} \\ 0_{d} \leq h \leq n_{d}}}\left(\omega_{n_{d}, h}\left(d_{x}^{4}, \mu\right)-\sum_{i, j=1}^{d}\left(\frac{h_{i}}{n}-x_{i}\right)^{2}\left(\frac{h_{j}}{n}-x_{j}\right)^{2}\right)=0
$$

uniformly w.r.t. $x \in Q_{d}$.

Then, for every $u \in C^{2}\left(Q_{d}\right)$,

$$
\lim _{n \rightarrow \infty} n\left(M_{n, \mu}(u)-u\right)=\sum_{i, j=1}^{d} \alpha_{i j} \frac{\partial^{2} u}{\partial x_{i} \partial x_{j}}+\sum_{i=1}^{d} \beta_{i} \frac{\partial u}{\partial x_{i}} \quad \text { uniformly on } Q_{d},
$$

where, for every $x \in Q_{d}$,

$$
\alpha_{i j}(x):=\gamma_{i j}(x)-x_{i} \beta_{j}(x)-x_{j} \beta_{i}(x) .
$$

Proof. On account of Theorem 1.5.2 in [4], (4.5) will be proved once we show that, for every $i, j=1, \ldots, d$,

(a) $\lim _{n \rightarrow \infty} n M_{n, \mu}\left(p r_{i} \circ \Psi_{x}\right)-\beta_{i}=0$ uniformly on $Q_{d}$;

(b) $\lim _{n \rightarrow \infty} n M_{n, \mu}\left(\left(p r_{i} \circ \Psi_{x}\right)\left(p r_{j} \circ \Psi_{x}\right)\right)-2 \alpha_{i j}=0$ uniformly on $Q_{d}$; 
(c) $\sup _{n \geq 1, x \in Q_{d}} n M_{n, \mu}\left(d_{x}^{2}\right)(x)<+\infty$;

(d) $\lim _{n \rightarrow \infty} n M_{n, \mu}\left(d_{x}^{4}\right)(x)=0$ uniformly w.r.t. $x \in Q_{d}$.

In order to prove statement (a), we first observe that, for any $n \geq 1, h \in \mathbf{N}^{d}$, $0 \leq h \leq n_{d}$ and $x \in Q_{d}$,

$$
\omega_{n_{d}, h}\left(\left(p r_{i} \circ \Psi_{x}\right), \mu\right)=\omega_{n_{d}, h}\left(p r_{i}, \mu\right)-x_{i} ;
$$

this, together with assumption (4.2), completes the proof.

We pass now to prove statement (b). It is, indeed, a consequence of (4.2) and (4.3), once one notices that

$$
\begin{aligned}
& \omega_{n_{d}, h}\left(\left(p r_{i} \circ \Psi_{x}\right)\left(p r_{j} \circ \Psi_{x}, \mu\right)=\left(\omega_{n_{d}, h}\left(p r_{i} p r_{j}, \mu\right)-x_{i} x_{j}\right)\right. \\
& -x_{i}\left(\omega_{n_{d}, h}\left(p r_{j}, \mu\right)-x_{j}\right)-x_{j}\left(\omega_{n_{d}, h}\left(p r_{i}, \mu\right)-x_{i}\right) .
\end{aligned}
$$

Statement (c) follows directly from (4.1).

Finally, we have to prove statement (d). We recall that for the sequence $\left(B_{n}\right)_{n \geq 1}$ of the Bernstein operators (3.4), one has that

$$
\lim _{n \rightarrow \infty} n B_{n}\left(d_{x}^{4}\right)(x)=0
$$

uniformly w.r.t. $x \in Q_{d}$ (see [2, Formula (5), p. 434 and Proposition 6.2.3]).

Under assumption (4.4), as for every $x \in Q_{d}$,

$$
\begin{aligned}
& n M_{n, \mu}\left(d_{x}^{4}\right)(x)=n B_{n}\left(d_{x}^{4}\right)(x)+n\left(M_{n, \mu}\left(d_{x}^{4}\right)(x)-B_{n}\left(d_{x}^{4}\right)(x)\right) \\
& \leq n B_{n}\left(d_{x}^{4}\right)(x)+n\left|M_{n, \mu}\left(d_{x}^{4}\right)(x)-B_{n}\left(d_{x}^{4}\right)(x)\right| \leq n B_{n}\left(d_{x}^{4}\right)(x) \\
& +n \sum_{\substack{h \in \mathbf{N}^{d} \\
0_{d} \leq h \leq n_{d}}}\left|\omega_{n_{d}, h}\left(d_{x}^{4}, \mu\right)-\sum_{i, j=1}^{d}\left(\frac{h_{i}}{n}-x_{i}\right)^{2}\left(\frac{h_{j}}{n}-x_{j}\right)^{2}\right|\left(\begin{array}{c}
n_{d} \\
h
\end{array}\right) x^{h}\left(1_{d}-x\right)^{n_{d}-h},
\end{aligned}
$$

we easily get that $\lim _{n \rightarrow \infty} n M_{n, \mu}\left(d_{x}^{4}\right)(x)=0$ uniformly w.r.t. $x \in Q_{d}$, and this finishes the proof.

Remark 4.2. As we have shown in (3.15), some estimates for the rate of convergence in (3.16), in terms of the $K$-functionals for $L^{p}$-spaces, are available. A more difficult question is to establish the rate of convergence of $M_{n, \mu}$ with respect to the uniform norm. Under the assumptions (i)-(ii) of Theorem 4.1, we can give a partial answer to this question.

From [10, Theorem 2], we infer the general estimate in terms of the second modulus of continuity $\omega_{2}(f, \delta)$ : for every $n \geq 1$ and $f \in C\left(Q_{d}\right)$,

$$
\left\|M_{n, \mu}(f)-f\right\|_{\infty} \leq C\left(\lambda_{n, \infty}\|f\|_{\infty}+\omega_{2}\left(f, \lambda_{n, \infty}^{1 / 2}\right)\right),
$$

where $C$ is an absolute constant depending only on $d, \lambda_{n, \infty}$ is defined by

$$
\begin{aligned}
& \lambda_{n, \infty}:=\max \left\{\left\|M_{n, \mu}(\mathbf{1})-\mathbf{1}\right\|_{\infty},\left\|M_{n, \mu}\left(p r_{1}\right)-p r_{1}\right\|_{\infty},\right. \\
& \left.\ldots,\left\|M_{n, \mu}\left(p r_{d}\right)-p r_{d}\right\|_{\infty},\left\|M_{n, \mu}\left(e_{2}\right)-e_{2}\right\|_{\infty}\right\},
\end{aligned}
$$

and $e_{2}:=\sum_{i=1}^{d} p r_{i}^{2}$. 
Assumption (i)-(ii) in Theorem 4.1 yield that there exists $M>0$ such that, for every $i=1, \ldots, d$

$$
\left\|M_{n, \mu}\left(p r_{i}\right)-p r_{i}\right\|_{\infty} \leq \frac{M}{n} \quad \text { and } \quad\left\|M_{n, \mu}\left(e_{2}\right)-e_{2}\right\|_{\infty} \leq \frac{M}{n} .
$$

Hence, since $M_{n, \mu}(\mathbf{1})=\mathbf{1}$, we have that

$$
\left\|M_{n, \mu}(f)-f\right\|_{\infty} \leq C\left(\frac{M}{n}+\omega_{2}(f, \sqrt{M / n})\right) .
$$

\section{An example}

In this last section, we discuss a concrete example where the previous results apply. We begin to choose the measure $\mu$. Let $a=\left(a_{1}, \ldots, a_{d}\right), b=\left(b_{1}, \ldots, b_{d}\right) \in \mathbf{R}^{d}$ such that $a_{i}>-1$ and $b_{i}>-1$ for any $i=1, \ldots, d$. As measure $\mu$ we consider the absolutely continuous measure $\mu_{a, b} \in M_{1}^{+}\left(Q_{d}\right)$ with respect to the Borel-Lebesgue measure $\lambda_{d}$ on $Q_{d}$ with density the normalized Jacobi weight

$$
w_{a, b}(x):=\frac{x^{a}(1-x)^{b}}{\int_{Q_{d}} y^{a}(1-y)^{b} d y} \quad\left(x \in \stackrel{\circ}{Q_{d}}\right) .
$$

Note that $\mu_{a, b}$ satisfies property (ii) in Theorem 3.1 .

In such a case, the operators $M_{n, \mu_{a, b}}$ turn into the so-called Bernstein-Durrmeyer operators on $Q_{d}$ with Jacobi weights, which were introduced and studied in [3]. More precisely, they are defined, for every $n \geq 1, f \in L^{1}\left(Q_{d}, \mu_{a, b}\right)$ and $x \in Q_{d}$, by setting

$$
M_{n}(f)(x):=\sum_{\substack{h \in \mathbf{N}^{d} \\
0_{d} \leq h \leq n_{d}}} \omega_{n_{d}, h}(f)\left(\begin{array}{c}
n_{d} \\
h
\end{array}\right) x^{h}\left(1_{d}-x\right)^{n_{d}-h}
$$

where, for every $n \geq 1$ and $h=\left(h_{1}, \ldots h_{d}\right) \in \mathbf{N}^{d}, 0_{d} \leq h \leq n_{d}$,

$$
\begin{aligned}
& \omega_{n_{d}, h}(f):=\frac{1}{\int_{Q_{d}} y^{h+a}\left(1_{d}-y\right)^{n_{d}-h+b} d y} \int_{Q_{d}} y^{h+a}\left(1_{d}-y\right)^{n_{d}-h+b} f(y) d y \\
& =\prod_{i=1}^{d} \frac{\Gamma\left(n+a_{i}+b_{i}+2\right)}{\Gamma\left(h_{i}+a_{i}+1\right) \Gamma\left(n-h_{i}+b_{i}+1\right)} \int_{Q_{d}} y^{h+a}\left(1_{d}-y\right)^{n_{d}-h+b} f(y) d y
\end{aligned}
$$

and $\Gamma(u)(u \geq 0)$ denotes the classical Euler Gamma function.

The operators $M_{n, \mu_{a, b}}$ satisfy assumptions (4.2)-(4.4); in particular, they verify the following asymptotic formula: for every $u \in C^{2}\left(Q_{d}\right)$,

$$
\lim _{n \rightarrow \infty} n\left(M_{n, \mu_{a, b}}(u)-u\right)=A(u) \quad \text { uniformly on } Q_{d},
$$

where the differential operator $A$ is defined by

$$
A(u)(x)=\sum_{i=1}^{d} x_{i}\left(1-x_{i}\right) \frac{\partial^{2} u}{\partial x_{i}^{2}}(x)+\left(a_{i}+1-\left(a_{i}+b_{i}+2\right) x_{i}\right) \frac{\partial u}{\partial x_{i}}(x)
$$

for every $u \in C^{2}\left(Q_{d}\right)$ and $x=\left(x_{1}, \ldots, x_{d}\right) \in Q_{d}$. 
Note that such a differential operator falls into the category of so-called FlemingViot operators. In [3], the authors proved that the operator $A$ is closable and its closure (pre)-generates a Markov semigroup $(T(t))_{t \geq 0}$ on $C\left(Q_{d}\right)$ such that, if $f \in C\left(Q_{d}\right)$ and $t \geq 0$, then

$$
T(t)(f)=\lim _{n \rightarrow \infty} M_{n}^{[n t]}(f) \text { uniformly on } Q_{d},
$$

$[n t]$ denoting the integer part of $n t(n \geq 1)$.

A similar relation holds true also in $L^{p}\left(Q_{d}, \mu_{a, b}\right)$. An open problem should be to understand under which conditions this holds true in the more general context of the Bernstein-Durrmeyer operators $M_{n, \mu}$ with respect to arbitrary measures.

\section{References}

[1] Albanese, A.A., Campiti, M., Mangino, E.M., Regularity properties of semigroups generated by some Fleming-Viot type operators, J. Math. Anal. Appl., 335(2007), 1259-1273.

[2] Altomare, F., Campiti, M., Korovkin-Type Approximation Theory and its Applications, de Gruyter Studies in Mathematics, 17, Walter de Gruyter, Berlin-New York, 1994.

[3] Altomare, F., Cappelletti Montano, M., Leonessa, V., On the positive semigroups generated by Fleming-Viot type differential operators, Comm. Pure Appl. Anal., 18(1)(2019), 323-340.

[4] Altomare, F., Cappelletti Montano, M., Leonessa, V., Raşa, I., Markov Operators, Positive Semigroups and Approximation Processes, de Gruyter Studies in Mathematics, 61, Walter de Gruyter GmbH, Berlin/Boston, 2014.

[5] Bauer, H., Probability Theory, de Gruyter Studies in Mathematics, 23, Walter de Gruyter \& Co., Berlin, 1996.

[6] Berdysheva, E.E., Uniform convergence of Bernstein-Durrmeyer operators with respect to arbitrary measure, J. Math. Anal. Appl., 394(2012), 324-336.

[7] Berdysheva, E.E., Bernstein-Durrmeyer operators with respect to arbitrary measure, II: Pointwise convergence, J. Math. Anal. Appl., 418(2014), 734-752.

[8] Berdysheva, E.E., Jetter, K., Multivariate Bernstein Durrmeyer operators with arbitrary weight functions, J. Approx. Theory, 162(2010), 576-598.

[9] Berdysheva, E.E., Li, B.Z., On $L^{p}$-convergence of Bernstein Durrmeyer operators with respect to arbitrary measure, Publ. Inst. Math., Beograd (N.S.), 96(110)(2014), 23-29.

[10] Berens, H., De Vore, R., Quantitative Korovkin Theorems for positive linear operators on $L_{p}$-spaces, Trans. Amer. Math. Soc., 245(1978), 349-361.

[11] Berens, H., Xu, Y., On Bernstein-Durrmeyer polynomials with Jacobi weights, in: C.K. Chui (ed.), Approximation Theory and Functional Analysis, Academic Press, Boston, 1991, 25-46.

[12] Derriennic, M.M., Sur l'approximation de fonctions intégrables sur [0,1] par des polynômes de Bernstein modifies, J. Approx. Theory, 31(1981), 325-343.

[13] Ditzian, Z., Multidimensional Jabobi-type Bernstein-Durrmeyer operators, Acta Sci. Math., Szeged, 60(1995), 225-243.

[14] Ditzian, Z., Totik, V., Moduli of Smoothness, Springer Series in Computational Mathematics, 9, Springer-Verlag, New York, 1987. 
[15] Durrmeyer, J.L., Une formule d'inversion de la transformée de Laplace: applications à la théorie des moments, Thèse de 3e cycle, Faculté des Sciences de l'Université de Paris, 1967.

[16] Lorentz, G.G., Bernstein Polynomials, University of Toronto Press, Toronto, 1953.

[17] Li, B.Z., Approximation by multivariate Bernstein-Durrmeyer operators and learning rates of least-squares regularized regression with multivariate polynomial kernels, J. Approx. Theory, 173(2013), 33-55.

[18] Lupaş, A., Die Folge der Betaoperatoren, Dissertation, Universität Stuttgart, 1972.

[19] Păltănea, R., Sur un opérateur polynomial défini sur l'ensemble des fonctions intégrables, Univ. Babeş-Bolyai, Cluj-Napoca, 83(1983), no. 2, 101-106.

[20] Royden, H.L., Real Analysis, third ed., Macmillan Publishing Company, New York, 1988.

[21] Vladislav, T., Raşa, I., Analiză Numerică, Aproximare, Problema lui Cauchy Abstractă, Proiectorii Altomare, Ed. Tehnică, Bucureşti, 1999.

[22] Waldron, Sh., A generalization of beta integral and the limit of Bernstein-Durrmeyer operator with Jacobi weights, J. Approx. Theory, 122(2003), 141-150.

Mirella Cappelletti Montano

Università degli Sudi di Bari Aldo Moro

Dipartimento di Matematica

Via E. Orabona 4

70125 Bari, Italy

e-mail: mirella.cappellettimontano@uniba.it

Vita Leonessa

Università degli Studi della Basilicata

Dipartimento di Matematica, Informatica ed Economia

Viale dell'Ateneo Lucano 10

85100 Potenza, Italy

e-mail: vita.leonessa@unibas.it 